\title{
Reflets
}

Revue ontaroise d'intervention sociale et communautaire

\section{L'impact du conflit armé sur l'intégration des femmes immigrantes et réfugiées francophones en Ontario}

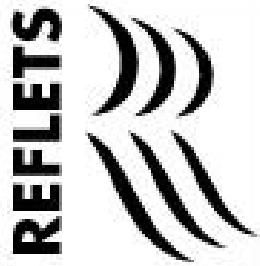

\section{Nathalie Plante, Angèle Bassole, Hoori Hamboyan, Michèle Kérisit et Marta \\ Young}

Volume 11, numéro 1, 2005

Exclusion sociale

URI : https://id.erudit.org/iderudit/013064ar

DOI : https://doi.org/10.7202/013064ar

Aller au sommaire du numéro

Éditeur(s)

Reflets : Revue ontaroise d'intervention sociale et communautaire

ISSN

1203-4576 (imprimé)

1712-8498 (numérique)

Découvrir la revue

Citer cet article

Plante, N., Bassole, A., Hamboyan, H., Kérisit, M. \& Young, M. (2005). L'impact

du conflit armé sur l'intégration des femmes immigrantes et réfugiées

francophones en Ontario. Reflets, 11(1), 178-186.

https://doi.org/10.7202/013064ar

Tous droits réservés (C) Reflets : Revue ontaroise d'intervention sociale et communautaire, 2005
Ce document est protégé par la loi sur le droit d'auteur. L'utilisation des services d'Érudit (y compris la reproduction) est assujettie à sa politique d'utilisation que vous pouvez consulter en ligne.

https://apropos.erudit.org/fr/usagers/politique-dutilisation/ 


\section{L'impact du conflit armé sur l'intégration des femmes immigrantes et réfugiées francophones en Ontario}

Nathalie Plante, Angèle Bassole, Hoori Hamboyan, Michèle Kérisit et Marta Young

Le Mouvement ontarien des femmes immigrantes

francophones (MOFIF)

C'est au printemps de 2003 qu'a débuté pour nous, chercheures, une expérience transformatrice: le Mouvement ontarien des femmes immigrantes francophones (MOFIF), encadré par un regroupement d'organismes communautaires de Toronto, de Hamilton et d'Ottawa ainsi que de femmes ayant vécu la guerre, initiait une recherche intitulée "L'impact du conflit armé sur l'intégration des femmes immigrantes et réfugiées francophones en Ontario ». Nous devions mener cette recherche et ne savions pas si les femmes ayant vécu la guerre dans leur pays d'origine allaient vouloir partager avec nous leurs expériences. Nous avons été vraiment touchées par leur besoin de parler, de raconter, de partager leur histoire afin que d'autres femmes ayant aussi vécu des conflits armés et se trouvant maintenant au Canada puissent bénéficier de leurs expériences et ainsi s'intégrer plus facilement à leur société d'accueil.

Nous voulons dans cet article rendre compte des recommandations qui ont émané d'abord de nos rencontres avec des femmes, 23 au total, ainsi que de groupes focus auprès 
d'intervenantes et d'intervenants travaillant avec des femmes ayant vécu des conflits armés. Ces recommandations, qui se trouvent dans le rapport de recherche ${ }^{1}$, ont aussi été retravaillées lors d'un forum de validation de la recherche réunissant plus de 60 participantes, soit des représentantes d'organismes communautaires et des femmes de la communauté. Les recommandations que nous allons présenter ici portent davantage sur l'intervention directe alors que le rapport et le compte-rendu du forum de validation proposent aussi une série de recommandations portant sur le démarchage politique pour modifier, par exemple, les lois sur l'immigration et la réunification familiale, pour faire augmenter les prestations d'assistance sociale et faciliter la reconnaissance des diplômes. Notre souhait est que la voix de ces femmes soit entendue et ait un impact réel sur leur vie au Canada et sur l'intégration d'autres femmes comme elles.

\section{L'impact des conflits armés sur les femmes francophones survivantes de guerre}

À une exception près, les femmes rencontrées dans le cadre de cette recherche étaient originaires d'Afrique centrale (Rwanda, Burundi et République démocratique du Congo - RDC). C'est leur témoignage que nous relevons ici, car elles ont, pour la plupart, vécu des conflits caractérisés par une violence extrême et continue et par la perpétration d'atrocités inimaginables. Certaines sont, par exemple, rescapées du génocide rwandais.

L'impact causé par cette violence se vit sur différents plans, physique, psychologique ou affectif. Alors que certaines femmes rencontrées portaient les séquelles physiques des sévices vécus, dont des problèmes gynécologiques dus à des viols parfois répétés, toutes portaient des cicatrices psychologiques et affectives. Une souffrance profonde est ressentie face aux pertes personnelles, familiales et sociales. Pour beaucoup, un sentiment de culpabilité d'avoir survécu se conjugue avec une profonde solitude. Pour certaines aussi, la perte du pays natal, à travers les conflits et 
"La réunification

d'avec les membres de leur famille restés au pays est souvent difficile pour de multiples raisons, celle en particulier des barrières légales et financières imposées par la Loi sur l'immigration et la protection des réfugiés et son règlement ${ }^{2}$." l'exil, crée des blessures identitaires, une nostalgie très difficile à supporter.

Les parcours des femmes rencontrées sont jalonnés de deuils multiples et de séparations familiales. Des êtres chers n'ont pas survécu ou ont été assassinés, alors que d'autres ont disparu sans que l'on sache où ils sont : ils ne sont peut-être pas en sécurité, ils ont souvent besoin d'aide financière. La réunification d'avec les membres de leur famille restés au pays est souvent difficile pour de multiples raisons, celle en particulier des barrières légales et financières imposées par la Loi sur l'immigration et la protection des réfugiés et son règlement ${ }^{2}$. Leurs pertes se situent également sur le plan social. Alors que la plupart étaient des femmes actives et professionnellement impliquées dans leur propre société, elles doivent, une fois arrivées au Canada, "repartir à zéro ", comme dit l'une d'entre elles. Elles ont alors à négocier avec des aides gouvernementales, l'aide sociale par exemple, qui leur permettent à peine de survivre, cela, pendant longtemps, alors qu'elles ont envie et besoin de reprendre le cours de leur vie, d'assurer aux enfants qui les accompagnent une intégration réussie.

Plusieurs symptômes pouvant être reliés au syndrome posttraumatique, mais aussi à l'anxiété créée par leurs conditions de vie actuelle nous ont souvent été rapportés : cauchemars, colère, crises d'angoisse subites, réminiscences involontaires, troubles du sommeil, haute tension, migraines, trouble de la mémoire, difficulté de concentration. Cela a des conséquences directes sur l'intégration des femmes au Canada : elles se perdent dans les mesures administratives, elles oublient des rendez-vous, certaines éprouvent des difficultés d'apprentissage puisqu'elles ne peuvent se concentrer; le choc culturel, commun à l'ensemble des nouveaux arrivants au Canada, est exacerbé; les difficultés financières et les difficultés rencontrées dans les tentatives de réunification familiale maintiennent les femmes dans leur situation d'exilées. On est au Canada, mais aussi encore là-bas, même dix ans après. 


\section{Les obstacles dans la recherche d'aide}

"...il faut donc penser l'intervention autrement, accueillir la femme dans toute sa personne, en ne la réduisant pas à l'expérience de guerre. Il faut imaginer un lieu où les femmes peuvent avoir la possibilité de parler de ce qu'elles ont vécu sans nécessairement que ce soit dans un cadre thérapeutique. "
Malgré tout, selon les intervenantes et intervenants rencontrés ainsi que selon certaines des femmes ayant participé à la recherche, les survivantes de guerre ne consultent pas pour de l'aide psychologique à moins de circonstances extrêmes, lorsqu'elles sont vraiment au pied du mur. Cela, pour diverses raisons : elles ne sont pas accoutumées à ce genre de services, elles ne savent pas qu'ils existent ou ne souhaitent pas les utiliser; elles ne sont pas prêtes à parler ou l'ont déjà fait et se sont senties jugées, elles ont senti que la personne à qui elles s'adressaient n'était pas prête à recevoir leurs propos. Pour les rejoindre, il faut donc penser l'intervention autrement, accueillir la femme dans toute sa personne, en ne la réduisant pas à l'expérience de guerre. Il faut imaginer un lieu où les femmes peuvent avoir la possibilité de parler de ce qu'elles ont vécu sans nécessairement que ce soit dans un cadre thérapeutique. Le lien de confiance établi avec une intervenante ou une personne significative, que ce soit dans le cadre de la recherche d'emploi par exemple ou d'une activité organisée à d'autres fins, est essentiel pour que les femmes puissent se raconter. Le temps nécessaire à la création de ce lien de confiance, les références trop rapides à d'autres intervenants « spécialistes » dans tel ou tel domaine (santé mentale et autres) sont des obstacles à l'intervention auprès de ces femmes. En effet, elles sont ainsi amenées à répéter leur histoire plusieurs fois et ont chaque fois à la revivre. Elles se font référer à leur communauté, mais sans que celle-ci soit nécessairement outillée pour aider puisque fonctionnant avec peu de ressources. De plus, le recours à la communauté d'origine, si elle peut parfois aider, est délicat dans la mesure où celle-ci vit parfois les mêmes conflits qui ont, justement, mené à l'exil.

L'un des obstacles principaux que rencontrent donc les répondantes de notre recherche est évidemment la quasiinexistence de services spécialisés en français. Alors qu'il existe, à Toronto et à Ottawa, des services destinés aux personnes victimes de torture, ceux-ci ne sont pas dispensés en français, ou très peu. 


\section{Recommandations}

Il est donc important que ce que les femmes ont vécu dans leur pays d'origine, l'impact que cela a eu sur elles et les conséquences pour celles-ci au Canada soient bien compris par les divers intervenantes et intervenants appelés à agir auprès d'elles.

\section{La sensibilisation et la formation des intervenantes et intervenants}

Ainsi, nous recommandons qu'au niveau municipal soit lancée une campagne de formation et de sensibilisation auprès des représentantes et des représentants du gouvernement (agents de police, Ontario au travail, etc.) concernant l'expérience des réfugiées, en particulier des survivantes de violence organisée. Nous recommandons aux agences participant au projet de voir à la sensibilisation et à la formation de leurs propres intervenantes afin d'assurer l'écoute des survivantes (si possible dans leur langue) en plus d'améliorer la formation d'autres intervenants et intervenantes dans la communauté.

\section{La concertation entre intervenantes et intervenants}

Afin d'éviter aux femmes d'avoir à relater leur expérience à chaque rencontre avec une nouvelle ou un nouvel intervenant et afin d'éviter le ballottement d'un service à l'autre, nous recommandons le développement d'une approche multidisciplinaire axée sur l'intégration des nouveaux et des nouvelles arrivantes: que les intervenantes et intervenants travaillent en collaboration avec la personne et non de manière séparée. Dans ce but, les communautés auxquelles appartiennent les femmes pourraient faciliter le réseautage entre les organismes venant en aide aux femmes immigrantes et réfugiées.

Plusieurs intervenantes ont souligné leur besoin de soutien dans leurs activités auprès des femmes ayant vécu la guerre. Il leur est difficile de se retrouver parfois seules à porter le poids des 
"Les intervenantes soulignent donc un besoin de formation spécifique par rapport à l'intervention auprès de ces femmes, mais aussi un besoin de soutien de la part de leur employeur et d'autres collègues travaillant aussi auprès de personnes, de femmes, ayant vécu la guerre. » confidences reçues, de se trouver impuissantes à aider, à soulager. Les intervenantes soulignent donc un besoin de formation spécifique par rapport à l'intervention auprès de ces femmes, mais aussi un besoin de soutien de la part de leur employeur et d'autres collègues travaillant aussi auprès de personnes, de femmes, ayant vécu la guerre. Afin d'outiller le plus possible les intervenantes et intervenants, nous recommandons donc au MOFIF de favoriser la diffusion des outils d'intervention qui seront développés dans le cadre de ce projet auprès de toutes les agences francophones en Ontario et de participer à la formation des survivantes de guerre pour que celles-ci soient en mesure d'offrir la formation et de sensibiliser les organismes à cette problématique. Les institutions de formation des intervenantes et intervenants sociaux et professionnels de la santé devraient également intégrer l'intervention auprès des victimes de guerre à leurs programmes de formation interculturelle. Dans ce cas, les survivantes de guerre devraient prendre une part active à l'élaboration des outils d'intervention.

Afin de favoriser le partage des connaissances et de l'expérience entre intervenantes et intervenants, d'éviter l'épuisement professionnel chez ceux-ci, afin de leur fournir le soutien nécessaire, des groupes de travail et d'intervention en français pourraient être mis en place dans chaque ville concernée, selon le contexte. Dans cette optique, nous recommandons que les gouvernements municipaux soutiennent financièrement la constitution de tels groupes de travail facilitant la concertation et la formation des intervenants et des intervenantes travaillant auprès de femmes survivantes de violence organisée.

\section{La mise en place de services de qualité en français}

Afin d'éviter autant que possible la revictimisation des femmes dans le processus de demande d'asile, nous recommandons que soient exercées des pressions pour que les femmes puissent avoir accès à des avocats francophones spécialisés dans la préparation à l'audience auprès de la Commission de l'immigration et du statut de réfugié et dans la défense des droits des femmes réfugiées. 
Pour que l'aide soit plus accessible, nous recommandons que soit créée une ligne de soutien spécifique pour les femmes survivantes de guerre et que les gouvernements municipaux soutiennent financièrement la création d'une telle ligne de soutien. Nous recommandons aussi que soit établie une base de données de services, cela, avec la participation du MOFIF.

Les femmes nous ont rapporté des expériences bien diverses de leur arrivée à la frontière canadienne : d'une prise en charge complète pour celles qui arrivent avec le statut de réfugiées déjà accordé selon la Convention de Genève, à un abandon complet pour celles qui en font la demande à la frontière. Pour ces dernières, à moins d'avoir de la famille où des amis au Canada chez qui se rendre ou venant les accueillir, ce que les expériences relatées avaient le plus souvent en commun c'était la "chance" d'avoir rencontré un étranger ou une étrangère qui a bien voulu les aider. De plus, les femmes rencontrées n'ont pas été mises systématiquement au courant des services d'accueil, qui existent pourtant : elles ont été laissées à elles-mêmes, parfois avec des enfants, sans un sou et sans savoir où aller. Nous recommandons donc au gouvernement fédéral d'établir une structure d'accueil (aux frontières et dans la communauté) plus concertée (entre les instances officielles et les groupes communautaires) et plus sécuritaire pour les personnes réfugiées arrivant aux frontières et dans les refuges; de mettre l'accent sur la constance et la qualité des services à l'accueil : que toutes les arrivantes reçoivent de l'information et que cette information soit identique pour toutes; et de mettre un numéro 1-800 à la disposition des femmes réfugiées afin qu'elles puissent obtenir, dès leur arrivée, de l'information et des références en français.

Au plan communautaire, nous recommandons l'élaboration de fiches d'information qui permettront aux personnes dans les communautés de connaitre les services en français ou les divers intervenants qui peuvent aider dans le processus d'immigration et d'intégration, par exemple, de bons avocats et des personnes clés dans les écoles. Les agences participant au projet pourraient assurer la diffusion de ces informations. 


\section{Conclusion : mettre l'accent sur l'accès à des services en français}

De façon générale, les femmes ont souligné un manque de services en français et un manque d'information sur les services disponibles. Plus précisément, certaines ont mentionné que l'utilisation souvent obligée, faute de services en français, de services de santé ou juridiques en anglais les plaçait dans une situation particulièrement difficile lorsqu'elles avaient à aborder des sujets délicats et intimes tels que les viols et les traumatismes vécus ainsi que les conséquences gynécologiques qui s'ensuivent. D'autre part, il nous a été signalé que la présence d'un interprète nuit à la communication, surtout quand on ne connait ni ses antécédents ni sa formation, surtout en matière de confidentialité.

Il est donc nécessaire que le gouvernement ontarien fasse connaitre de façon systématique les services en français. D'autre part, les services existants de soutien aux personnes victimes de violence organisée se doivent d'augmenter et de développer leurs programmes en français pour que les femmes traumatisées par la guerre et leur famille puissent avoir accès aux mêmes services que ceux offerts aux personnes de langue anglaise. L'utilisation d'interprètes devrait être balisée par une formation appropriée, tant auprès des interprètes eux-mêmes qu'auprès des intervenantes et intervenants qui ont recours à leurs services. Cela nous semble particulièrement important en raison de la nature des conflits extrêmement violents qui ont cours en Afrique centrale. Enfin, nous recommandons aux communautés auxquelles appartiennent les femmes de se familiariser avec les ressources en santé mentale offertes dans chaque ville et de s'assurer que des services y soient offerts en français.

Naturellement, cela n'est qu'un bref aperçu de certains résultats de la recherche se rapportant davantage à l'intervention directe. Pour terminer, signalons que cette recherche a eu des suites dans le cadre desquelles vous pouvez vous inscrire. En effet, une nouvelle trousse de sensibilisation et de formation est en cours 
d'élaboration et sera sous peu disponible au MOFIF. Financée par l'Office des affaires francophones de l'Ontario et la fondation Trillium, «Prévenir la revictimisation des femmes francophones survivantes de conflits armés » est destinée aux intervenantes et aux intervenants sociaux, aux professionnelles et aux professionnels de la santé ainsi qu'au ministère de la Citoyenneté et Immigration. De plus, un réseau des intervenantes et intervenants francophones hors Québec sera aussi mis sur pied grâce au soutien financier du CNFS, volet Recherche de l'Université d'Ottawa. Ainsi, nous espérons que les témoignages des participantes à cette recherche auront un impact concret sur la façon dont est pensée l'intervention leur étant destinée.

\section{Notes}

1 Le rapport de recherche est disponible sur le site Internet de l'Action ontarienne contre la violence faite aux femmes http://francofemmes.org/aocvf/ ou en contactant directement le MOFIF au (613) 241-2128.

2 Gazette du Canada, édition spéciale,Vol. 136, n 9, Ottawa, juin 2002 